\title{
ARTí́CULO
}

\section{Macroinfauna en playas arenosas de la costa del Norte Grande de Chile sometidas a diferentes presiones antrópicas}

Sandy beach macroinfauna from the coast of northern Chile affected by different anthropic pressures

\author{
Emilio O. Acuña ${ }^{1}$ y Eduardo Jaramillo²
}

${ }^{1}$ Calle Nueva 375, Valdivia, Chile

${ }^{2}$ Facultad de Ciencias, Campus Isla Teja, Universidad Austral de Chile, Valdivia, Chile. ejaramillo@uach.cl

\begin{abstract}
Urban sandy beaches are affected by artificial grooming and recreational activities that result in disturbances of sediments and macroinfauna. During October 2012, 3 urban and 4 non-urban beaches were sampled along the coast of northern Chile (ca., $20-21^{\circ} \mathrm{S}$ ), in order to evaluate their intertidal macroinfauna community structure. Four transects set - perpendicular to the shoreline - were replicated from the upper intertidal to the low tide level to collect sediment samples and macroinfauna on the upper, middle and lower beach. The amount of debris stranded across the shore was used as an estimation of beach grooming. Urban beaches with grooming (i.e., almost no debris) had lower species richness than nonurban beaches without grooming and thus with stranded debris (4-5 vs. 8-9 spp., respectively). The crab Ocypode gaudichaudii was absent in 2 urban beaches where artificial grooming is used; on the other hand, even when they are present in an urban beach with artificial grooming (Huayquique) the burrow's diameter of this crab were the lowest at that site. No significant differences were found in the population abundances of the other 2 common species occurring in urban and non-urban beaches: the isopod Excirolana braziliensis and the anomuran Emerita analoga. Thus, it is concluded that artificial grooming affects primarily to an upper shore species, the crab 0 . gaudichaudii, which is a conspicuous member of the faunal communities in sandy beaches of northern Chile.
\end{abstract}

Key words: Sandy beaches, northern Chile, anthropic disturbance

Resumen.- Las playas arenosas urbanas son afectadas por limpieza artificial y recreación, lo que resulta en perturbación de los sedimentos y la macroinfauna. Durante octubre 2012, se muestrearon 3 playas urbanas y 4 no urbanas a lo largo de la costa del norte de Chile (ca., $20-21^{\circ} \mathrm{S}$ ), para evaluar la estructura comunitaria de la macroinfauna intermareal. Se replicaron 4 transectos perpendiculares a la línea de costa extendidos entre el intermareal superior y el nivel de la marea baja, para recolectar muestras de sedimento y macroinfauna en los niveles superior, medio e inferior de la playa. La cantidad de basura varada en la zona de muestreo fue utilizada como un estimador de limpieza artificial. Las playas urbanas con limpieza artificial (i.e., basura casi ausente) tuvieron menor riqueza de especies que playas no urbanas sin limpieza y con presencia de basura varada (4-5 vs. 8-9 spp., respectivamente). El cangrejo (carismático) Ocypode gaudichaudii estuvo ausente en 2 playas urbanas donde se practica limpieza artificial; aun cuando presente en una playa urbana con limpieza (Huayquique), las madrigueras de 0 . gaudichaudii fueron las de menor diámetro. No se encontraron diferencias significativas en las abundancias poblacionales de las otras 2 especies más comunes que ocurren en playas urbanas y no urbanas del área: el isópodo Excirolana braziliensis y el anomuro Emerita analoga. Por lo tanto, la limpieza artificial en las playas del área afecta primariamente a una especie de la parte superior de la playa, el cangrejo 0. gaudichaudii, miembro conspicuo de la comunidad faunística de las playas arenosas del norte de Chile.

Palabras clave: Playas arenosas, norte de Chile, perturbación antrópica

\section{INTRODUCCIÓN}

Las playas arenosas alrededor del mundo (ca., $75 \%$ de la costa libre de hielo; Brown 2001) prestan servicios ecosistémicos, muchos de ellos fundamentales para mantener el uso que el ser humano hace de ellas (Nel et al. 2014). Entre tales servicios están la disipación de eventos extremos (tormentas, tsunamis), respuesta a los cambios en el nivel del mar, descomposición de materia orgánica, filtración y purificación de agua, turismo y recreación (Defeo et al. 2009). Las actividades relacionadas a recreación, limpieza artificial y contaminación de la zona costera ocurren muchas veces en zonas de alto atractivo turístico, como es el caso de las playas arenosas del 
extremo norte de Chile y que por su clima, atraen gran número de turistas en cualquier época del año (Municipalidad de Iquique) ${ }^{1}$. Una de las ciudades del norte de Chile con más turismo es Iquique, con playas que se encuentran dentro del radio urbano y que son generalmente limpiadas artificialmente, actividad que perturba las capas superficiales del sedimento (Fig. 1a); por el contrario, en aquellas playas afuera del radio urbano, es común encontrar varazones de basura inorgánica y orgánica (Fig. 1b).

Jaramillo (1987) y Jaramillo et al. (2000), estudiaron la abundancia y zonación de la macroinfauna intermareal en playas arenosas de la costa de Iquique, observando baja riqueza de especies en relación a otras playas del litoral chileno. En ellas, el crustáceo anomuro Emerita analoga (Stimpson, 1857) fue dominante en los niveles inferiores y el cangrejo Ocypode gaudichaudii (H. Milne Edwards $\&$ Lucas, 1843) en los niveles superiores. A diferencia de lo observado en playas del centro y sur de Chile, Jaramillo (1987) no encontró crustáceos anfípodos (Talitridae) en los niveles superiores de estas playas, y solo una especie de isópodo cirolánido Excirolana braziliensis (Richardson, 1912) en los niveles medios.

Los efectos de la perturbación de origen antrópico sobre la fauna de las playas arenosas, han sido escasamente estudiados alrededor del mundo. Veloso et al. (2006), estudiaron el efecto del desarrollo urbano en playas arenosas de la costa de Rio de Janeiro, Brasil, encontrando que en playas donde ocurre un mayor número de visitantes, las abundancias del isópodo cirolánido E. braziliensis, son menores a las observadas en playas con menor afluencia de visitantes. Similarmente, Vieira et al. (2012) encontraron efectos negativos en playas urbanas en el sur de Brasil, tanto en abundancia como riqueza especifica de la macroinfauna intermareal. Por otra parte, Aheto et al. (2011), sugieren que el tamaño de las madrigueras de Ocypode spp. en la costa de Ghana, es un buen indicador del impacto humano en playas arenosas expuestas al oleaje, observándose una menor densidad de madrigueras en playas con mayor perturbación mecánica. Los estudios de Schlacher et al. (2007), muestran efectos negativos del paso de vehículos sobre las poblaciones de Ocypode cordimanus (Latreille, 1818) y Ocypode ceratophthalma (Pallas, 1772) en playas de North Stradbroke Island, Australia; i.e., la abundancia de estas especies en playas con alto tráfico de vehículos es baja, estimándose que en el peor de los casos, un solo vehículo puede matar el $0,75 \%$ de la población, lo que con un tráfico menor a 100 vehículos por día podría causar la muerte del $50 \%$ de la población intermareal de estas especies de cangrejos.
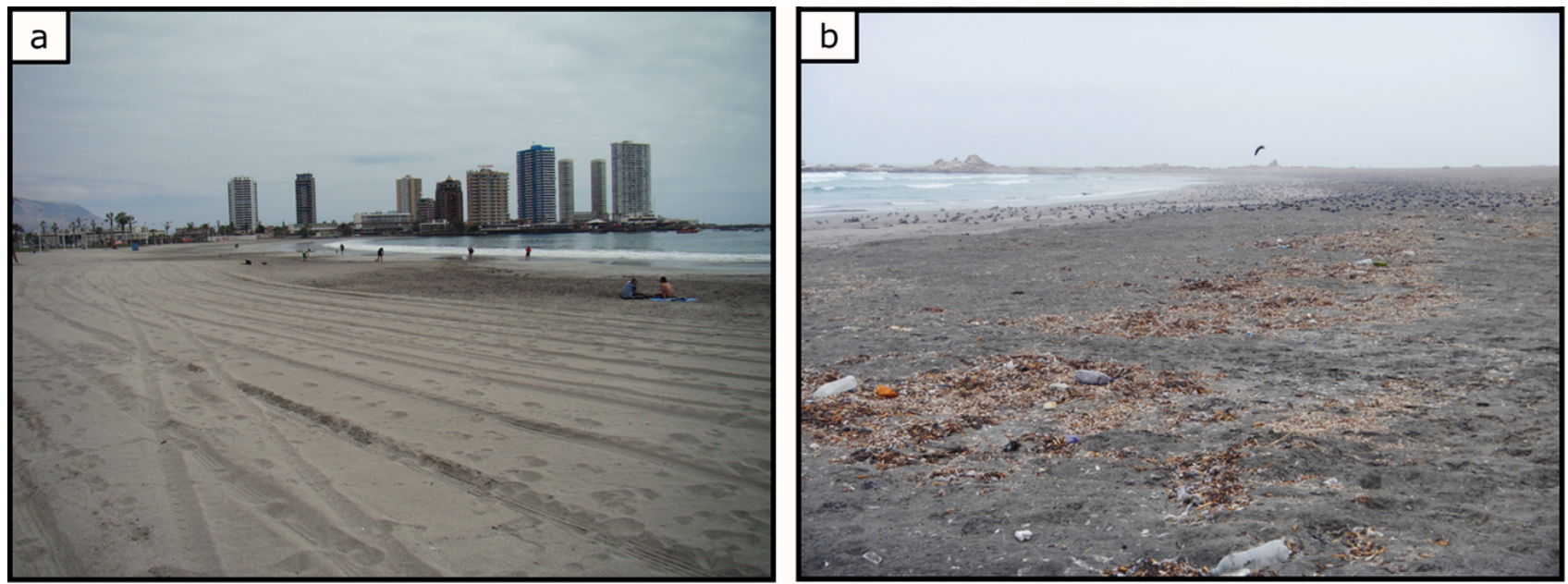

Figura 1. a) Cavancha, playa arenosa del centro urbano de Iquique. Nótese la perturbación de la arena producto de la limpieza de las mismas. b) Chomache, playa ubicada fuera del radio urbano de Iquique y donde no ocurre limpieza de la basura que se deposita periódicamente en los niveles superiores de la misma / a) Cavancha, an urban sandy beach of Iquique. Take note of the sand disturbance as a result of the artificial grooming of this site. b) Chomache, a non - urban beach without artificial grooming of the debris stranded on the upper shore levels

${ }^{1}<$ http://www.municipioiquique.cl/iquique/turismo-2/> 
El objetivo de este estudio fue comparar la abundancia y riqueza de especies de la macrofauna intermareal en playas ubicadas dentro y fuera del radio urbano de Iquique, a fin de evaluar eventuales efectos de la limpieza artificial que se realiza en las primeras sobre tales atributos comunitarios de esa macrofauna.

\section{Materiales y MÉTODOS}

\section{Área de ESTUdio}

Este estudio se llevó a cabo durante octubre 2012, en 7 playas arenosas, localizadas entre la ciudad de Iquique por el norte y el río Loa por el sur (Región de Tarapacá, ca., $\left.20-21^{\circ} \mathrm{S}\right)$. Tres de estas playas se ubican dentro del radio urbano de Iquique: Cavancha (20¹3'54,3”'S;7008'50,1 ' W), Playa Brava $\left(20^{\circ} 14 ' 28,7^{\prime \prime} \mathrm{S} ; 70^{\circ} 08^{\prime} 44,7^{\prime \prime} \mathrm{W}\right)$ y Huayquique (20¹6'51,2”S; $\left.70^{\circ} 07^{\prime} 44,3^{\prime \prime} \mathrm{W}\right)$, a la vez que 4 se ubican al sur del radio urbano de esta ciudad: Chauca (2049'24,3" $S$;

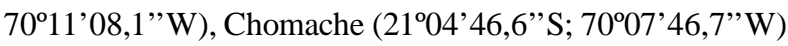
Boca del Diablo $\left(21^{\circ} 10^{\prime} 45,8^{\prime}\right.$ "S; $70^{\circ} 06$ ' 12,1 ' 'W) y Playa Larga (21 ${ }^{\circ} 17^{\prime} 46,3$ ”S; 7003'57,4”W) (Fig. 2).

\section{MUESTREO DE MACROINFAUNA Y CARACTERIZACIÓN FíSICA DE LAS PLAYAS}

Se muestreó sobre 4 transectos perpendiculares a la línea de costa, y separados $5 \mathrm{~m}$ entre sí. Los transectos se extendieron entre la línea de la marea alta más antigua hasta el límite de la marea baja. Cada transecto se dividió en 3 zonas: A) zona superior: extendida entre el límite de la marea alta más antigua y la marea alta más reciente; B) zona media: entre la marea alta más reciente y la línea de efluente, y C) zona inferior: entre la línea de efluente y el nivel de marea baja del día de muestreo. Las zonas A, B y

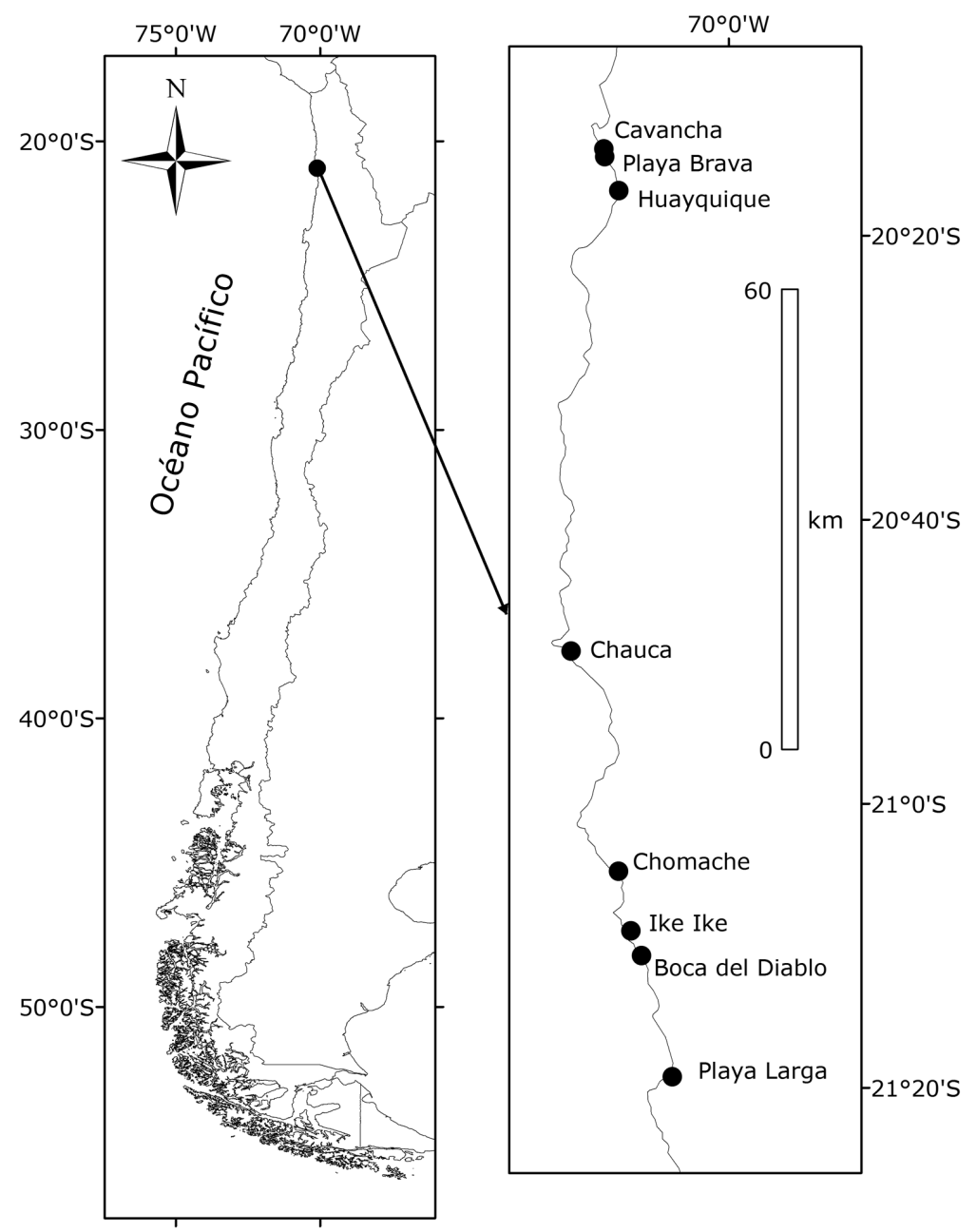

Figura 2. Mapa del área de estudio en la costa de Iquique, Chile. Los círculos negros indican la ubicación de los sitios de muestreo / Map of the study area at the coast of Iquique, Chile. The black dots indicate the location of the study sites 
C corresponden a las zonas de secado, retención y resurgencia respectivamente, definidas por Salvat (1964). En cada zona y a lo largo de los transectos, se recolectaron 5 muestras con un cilindro metálico (10 cm de diámetro) enterrado $20 \mathrm{~cm}$ en la arena. Estas 5 muestras se colocaron en un tamiz de abertura de malla de $1000 \mu \mathrm{m}$ para proceder a un proceso de filtración para separar la arena de los organismos de la macroinfauna. Los organismos retenidos en la malla se almacenaron en bolsas plásticas con formalina al $10 \%$ para su posterior análisis en laboratorio.

También se recolectaron muestras de sedimentos para análisis granulométricos en los siguientes niveles: marea alta más antigua, marea alta más reciente, línea de efluente y nivel de marea baja. En cada nivel se recolectaron cuatro muestras de los primeros $5 \mathrm{~cm}$ del sedimento y sobre los 4 transectos mencionados anteriormente. Estas muestras se analizaron mediante estudio de velocidades de sedimentación de las partículas de arena (Emery 1938) y método de momentos (Seward-Thompson \& Hails 1973). En cada playa se registró el ancho (medido desde el nivel de la marea alta antigua hasta el nivel de marea baja) y pendiente de la misma (Emery 1961). Esta última se expresa como $1 / x$, donde $x$ es la distancia en metros necesaria para tener un desnivel de $1 \mathrm{~m}$. Mientras mayor es el valor de $x$, más plana es una playa (i.e., menor declive o inclinación).
Para la estimación de la abundancia y distribución de Ocypode gaudichaudii, se contó la cantidad de agujeros en la playa ( $c f$., Quijón et al. 2001). Se muestreó sobre 6 transectos perpendiculares a la línea de costa, separados por $5 \mathrm{~m}$ entre si y extendidos entre la línea de marea alta más antigua y la línea de efluente; cada transecto tuvo un ancho de $2 \mathrm{~m}$. En cada transecto se determinó la cantidad total de madrigueras de Ocypode y el diámetro de las mismas.

\section{Perturbación del Sedimento}

Se obtuvieron 4 fotografías en el nivel de la marea alta más antigua, nivel de marea alta del día y línea de efluente, niveles que corresponden a los de mayor acumulación de basura. Las fotografías se tomaron sobre áreas de $100 \mathrm{x}$ $100 \mathrm{~cm}$ (Fig. 3).

\section{ANÁLISIS DE LOS DATOS}

Los datos de abundancia de la macroinfauna fueron llevados a metro lineal (ind. $\mathrm{m}^{-1}$ ) para estandarizar los diferentes anchos intermareales de cada una de las playas de este estudio. Este se calculó multiplicando la cantidad de individuos de cada muestra, por un factor de conversión, el que considera el ancho de la zona dividido por el área de la muestra obtenida $\left(\mathrm{n}^{\circ}\right.$ de cores por el área de cada uno).

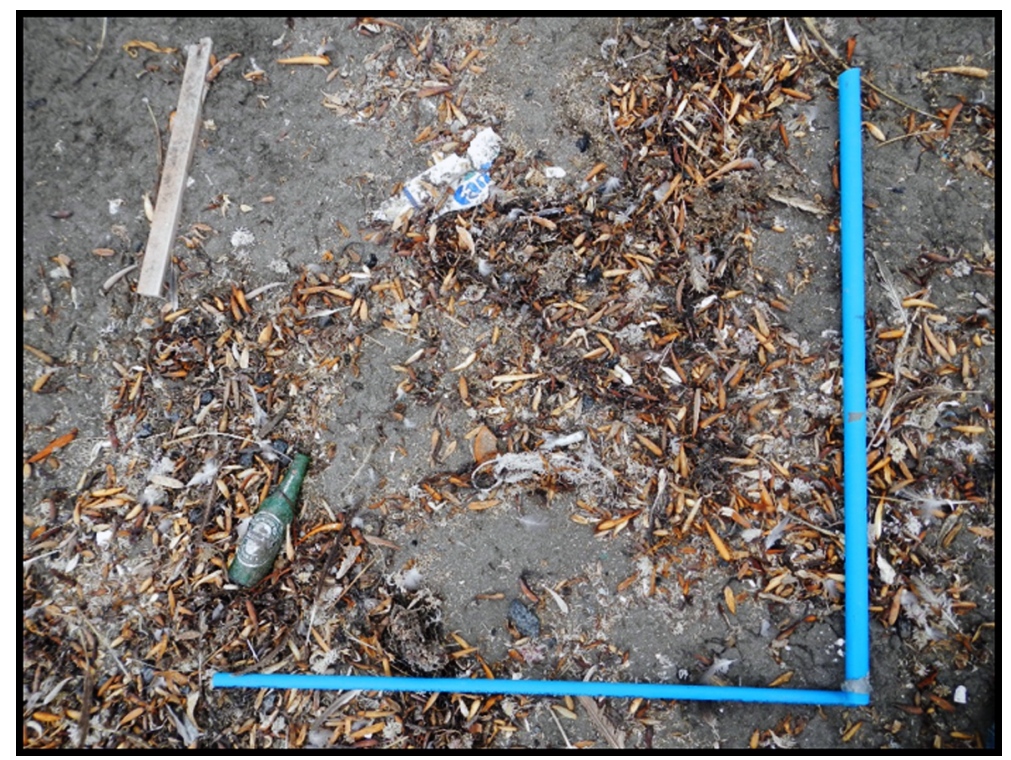

Figura 3. Fotografía de la cantidad de basura depositada en el nivel de marea alta antiguo en la playa de Chomache (3 octubre 2012). Cada vara plástica mide $100 \mathrm{~cm} /$ Stranded debris at the oldest high tide level of the beach Chomache (October 3, 2012). Each plastic rod measured $100 \mathrm{~cm}$ 
Para evaluar eventuales diferencias entre los tipos de playas, se utilizó el análisis no paramétrico de KruskalWallis (Kruskal \& Wallis 1952). Se realizaron análisis de correlación de Pearson (datos normales) y de Spearman (datos no normales) para analizar la relación de los parámetros comunitarios de la macroinfauna con su cercanía al centro urbano de Iquique.

Las fotografías destinadas a evaluar la cantidad de basura depositada en las playas, se analizaron con el programa CPCe 4.1 (Kohler \& Gill 2006). Se usaron 100 puntos distribuidos uniformemente sobre la fotografía, distinguiéndose las categorías basura y arena. Con estos datos se obtuvo un porcentaje total de basura para cada nivel, los que fueron promediados por cada nivel y playa, para calcular el contenido de basura por playa. La abundancia de basura fue utilizada como una estimación de la perturbación que se produce al realizar limpieza artificial en las playas estudiadas. Mientras más alto es el contenido de basura en la playa, menor es la perturbación por limpieza artificial en la misma.

\section{Resultados}

\section{Parámetros físicos}

\section{ANCHO de LA ZONA INTERMAREAL}

El ancho de la zona intermareal, varió entre $67 \mathrm{~m}$ en Huayquique y 159 m en Playa Larga. Las playas urbanas fueron, en general, más angostas $(67-74 \mathrm{~m})$ que aquellas ubicadas fuera del radio urbano (111-159 m) (Tabla 1).

\section{TAMAÑo MEdio de laS PaRTículas de ARENA}

El tamaño medio de las partículas de arena en el nivel superior de las playas (correspondiente a la marea alta más antigua), fluctuó entre 256 y $354 \mu \mathrm{m}$ (Playa Larga y Playa Brava); i.e., arenas de tamaño medio ( $c f$. ., Folk 1980) (Tabla 1). La variación entre los tamaños de partícula en el nivel de la marea alta de día o más reciente, fluctuó entre 228 y $356 \mu \mathrm{m}$. Playa Larga y Chauca presentaron los tamaños más bajos de partículas en este nivel (228 y 248 $\mu \mathrm{m}$, respectivamente), correspondientes a un tamaño de grano de arena fino ( $c f$. ., Folk 1980). En los otros sitios, el tamaño medio de la partícula de arena fluctuó entre 267 y $356 \mu \mathrm{m}$ (arenas de tamaño medio, 250-500 $\mu \mathrm{m} ; c f$., Folk 1980) (Tabla 1). El tamaño medio de las partículas de arena en la línea de efluente varió entre 236 y $333 \mu \mathrm{m}$ (Playa Larga y Playa Brava, respectivamente). En Playa Larga, se encontró el menor tamaño de partículas (236 $\mu \mathrm{m}$; arenas finas; $c f$., Folk 1980), mientras que en los otros sitios, este nivel estuvo caracterizado por arenas de tamaño medio (250-500 $\mu \mathrm{m} ; c f$., Folk 1980) (Tabla 1). El tamaño medio de las partículas de arena en el límite de la marea baja, fluctuó entre 277 y $437 \mu \mathrm{m}$ (Playa Larga y Playa Brava, respectivamente) correspondientes a arenas de tamaño medio ( $c f$. , Folk 1980) (Tabla 1).

\section{Pendiente de la playa}

Playa Brava fue la playa con la mayor inclinación o pendiente (1/25), a la vez que Chauca, Chomache y Playa Larga fueron las de menor inclinación (i.e., playas más planas) (1/48-1/58). Cavancha, Huayquique y Boca del Diablo tuvieron valores intermedios de pendiente (1/34-1/39) (Tabla 1).

Tabla 1. Ancho del intermareal, tamaño promedio de las partículas de arena en micrones y pendiente $(1 / x)(1$ desviación estándar en paréntesis). MAA=marea alta más antigua, $M A D=$ marea alta del día, $L E=$ =ínea de efluente y $\mathbf{M B}=$ nivel de marea baja / Intertidal width, mean size of sand particles in microns and beach face slope $(1 / \mathrm{x})$ ( 1 standard deviation in parentheses). $M A A=$ oldest high tide line, $M A D=$ high tide line of the sampling day, $L E=$ effluent line and $\mathrm{MB}=$ low tide line

\begin{tabular}{lcccccc}
\hline & \multirow{2}{*}{$\begin{array}{c}\text { Ancho del } \\
\text { intermareal }\end{array}$} & \multicolumn{4}{c}{ Tamaño de la partícula de arena $(\mu \mathrm{m})$} & \multirow{2}{*}{$\begin{array}{c}\text { Pendiente de } \\
\text { Playa }\end{array}$} \\
\cline { 5 - 6 } & $(\mathrm{m})$ & MAA & MAD & LE & MB & $(1 / \mathrm{x})$ \\
\hline Cavancha & 74 & $300(20)$ & $267(3)$ & $288(25)$ & $325(27)$ & $32(3,6)$ \\
Playa Brava & 68 & $354(29)$ & $291(17)$ & $333(9)$ & $437(37)$ & $25(0,5)$ \\
Huayquique & 67 & $292(10)$ & $313(5)$ & $277(10)$ & $326(16)$ & $34(2,2)$ \\
Chauca & 135 & $257(5)$ & $248(3)$ & $265(14)$ & $323(17)$ & $48(1,3)$ \\
Chomache & 135 & $307(9)$ & $356(16)$ & $316(10)$ & $325(10)$ & $50(4,2)$ \\
Boca del Diablo & 111 & $322(7)$ & $306(7)$ & $323(4)$ & $394(49)$ & $39(0,6)$ \\
Playa Larga & 159 & $256(5)$ & $228(2)$ & $236(2)$ & $277(4)$ & $58(0,5)$ \\
\hline
\end{tabular}




\section{RELACIÓN ENTRE PENDIENTE DE LA PLAYA Y TAMAÑO DE LA PARTÍCULA DE ARENA}

Los resultados de los análisis de correlación llevados a cabo para evaluar eventuales relaciones entre el tamaño medio general de la arena (i.e., promedio de los 3 niveles inferiores) y la pendiente, no mostraron correlación significativa entre ambas ( $\mathrm{r}$ de Pearson $=-0,63 ; P=0,13$ ).

\section{Parámetros biológicos}

\section{ZONACIÓN DE LA MACROINFAUNA}

La Tabla 2 y la Figura 4 dan cuenta de la zonación intermareal de la macroinfauna de las playas arenosas estudiadas. El coleóptero tenebriónido Phalerisida maculata (Kulzer) se recolectó solamente en la zona de secado (sensu Salvat 1964) de la playa, a la vez que el decápodo braquiuro Ocypode gaudichaudii y el isópodo cirolánido Excirolana braziliensis, se detectaron en las zonas de secado y retención (sensu Salvat 1964) en prácticamente todas las playas en que estas especies fueron observadas (Tabla 2). El decápodo anomuro Emerita analoga, se recolectó en la zona de resurgencia (sensu Salvat 1964) de todas las playas; en esta misma zona y en 3 de las playas, se recolectó el anomuro Lepidopa chilensis (Lenz, 1902). El braquiuro Bellia picta (H. Milne Edwards, 1848) solo apareció en la zona de resurgencia de Chauca (Tabla 2). Los poliquetos fueron los invertebrados que presentaron una distribución más variable; i.e., una especie de la familia Glyceridae, se detectó desde la zona de retención hasta la zona de resurgencia de la mayoría de las playas, a la vez que una especie de la familia Spionidae se recolectó tanto en la zona de retención como en la de resurgencia, mientras que una especie de la familia Nephtyidae y una especie de la familia Orbiniidae solo aparecieron en la zona más baja de la playa (i.e., zona de resurgencia) (Tabla 2).

\section{COMPOSICIÓN ESPECÍFICA Y ABUNDANCIAS DE LA ESPECIES DE LA MACROINFAUNA}

Los valores más altos de riqueza específica $(n=9$ especies), se registraron en Chomache y Playa Larga, mientras que los más bajos se detectaron en Playa Brava, Cavancha y Huayquique ( $n=4-5$ especies) (Tabla 3 ). Los resultados de los análisis de correlación realizados para evaluar la relación entre esta variable y la distancia de los sitios de muestreo al centro urbano de Iquique, mostraron una correlación significativa ( $\mathrm{r}$ de Pearson $=0,95 ; P=0,001$ ) (Fig. 5). Es decir, las playas más alejadas de ese centro (i.e., zona urbana) tienen un mayor complemento de especies que aquellas cercanas al centro. Los análisis efectuados (Kruskal-Wallis) para comparar la riqueza de la macroinfauna entre los 2 tipos de playas (urbanas con limpieza artificial y no urbanas sin esta perturbación), mostraron diferencias significativas entre ambos $(P<$ 0,001) (Fig. 5), con valores más altos en las segundas.

Tabla 2. Especies presentes en cada una de las zonas físicas de las playas estudiadas / Species inhabiting each of the physical zones at the beaches studied

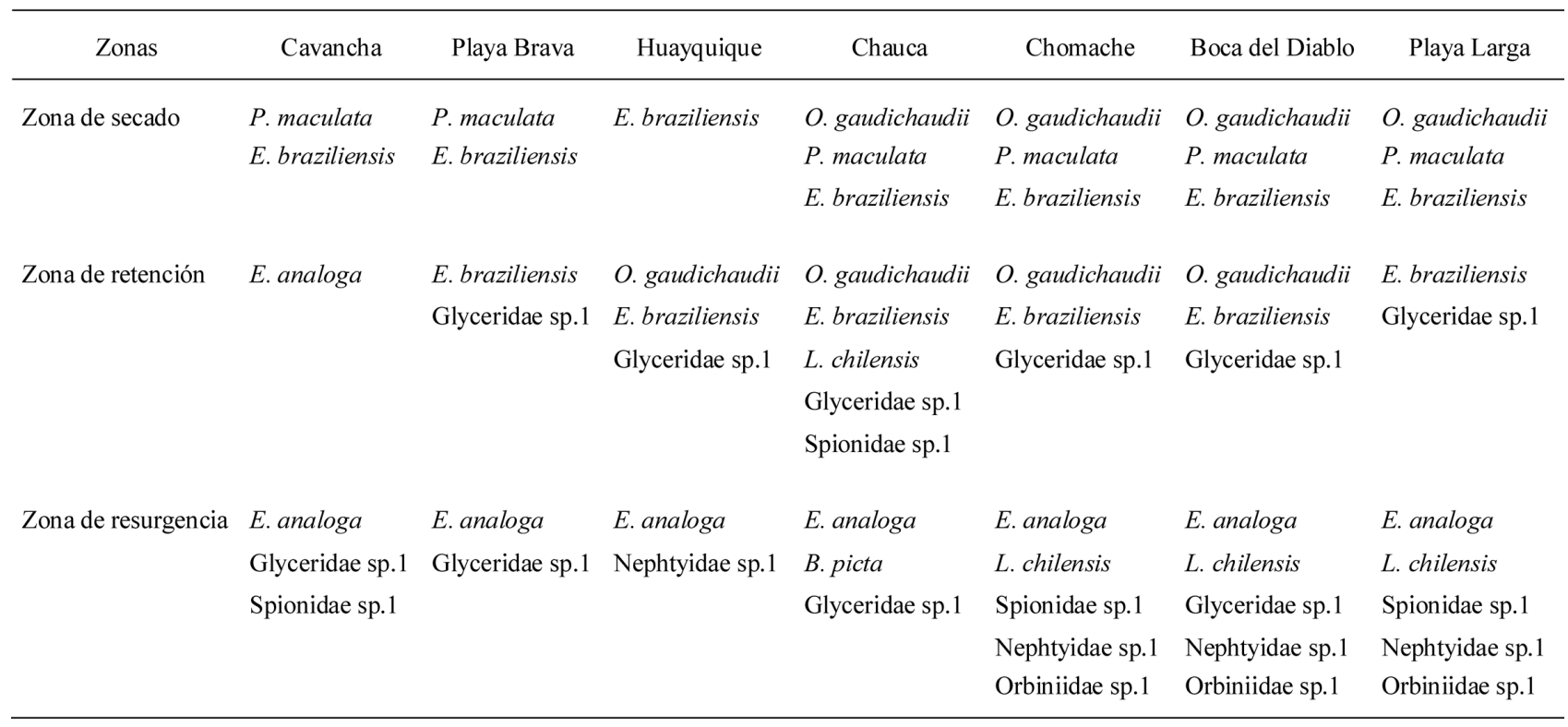




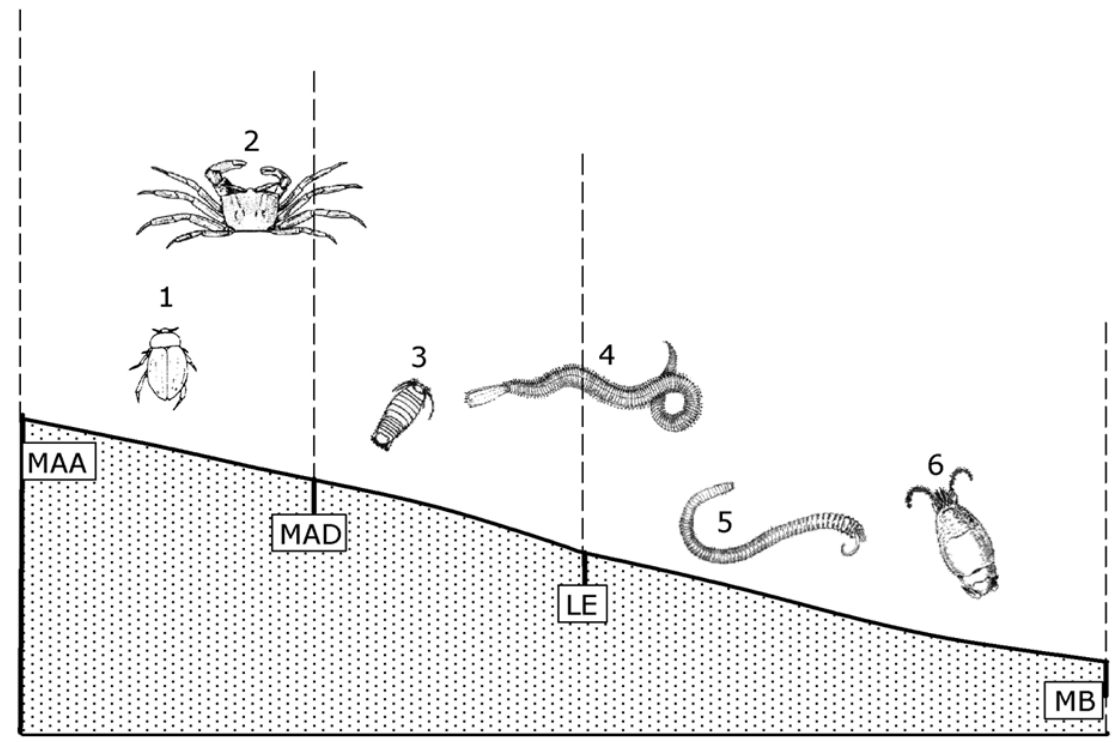

Figura 4. Distribución general de la macroinfauna que ocurre a lo ancho de las playas arenosas del área de estudio. MAA= marea alta antigua, $M A D=$ marea alta del día, $L E=$ línea de efluente y $M B=$ nivel de marea baja. Para cada zona de la playa se muestran las especies características de las mismas. 1: Phalerisidia maculata, 2: Ocypode gaudichaudii, 3: Excirolana braziliensis, 4: Gliceridae sp., 5: Spionidae sp., 6: Emerita analoga / General across shore distribution of the sandy beach macroinfauna occurring at the sandy beaches of the study area. $M A A=$ oldest high tide line, $M A D=$ high tide line of the sampling day, $\mathrm{LE}=$ effluent line and $\mathrm{MB}=$ low tide line. The characteristic species for each zone are shown: 1: Phalerisidia maculata, 2: Ocypode gaudichaudii, 3: Excirolana braziliensis, 4: Gliceridae sp., 5: Spionidae sp., 6: Emerita analoga

Tabla 3. Riqueza específica, promedios de abundancia total y abundancias de las especies de la macroinfauna en las playas estudiadas. Los valores se expresan en $\mathrm{m}^{-1}$ para la abundancia total y para todas las especies con excepción de 0 . gaudichaudii, para la cual los valores se expresan en $\mathbf{~}^{2}$. Por lo tanto, los valores de abundancia total no incluyen a este cangrejo / Species richness, mean abundances of the total macroinfauna and that of each species at the beaches studied. The values are expressed in $\mathrm{m}^{-1}$ for all the species but 0 . gaudichaudii whose abundances are expressed in $\mathrm{m}^{2}$. Thus, total abundances do not include abundances of this crab

\begin{tabular}{lrrrrrrr}
\hline & CAV & PB & HUA & CHA & CHO & BD & PL \\
\hline Riqueza específica & \multicolumn{1}{c}{5} & \multicolumn{1}{c}{4} & \multicolumn{1}{c}{5} & \multicolumn{1}{c}{8} & 9 & 8 & 9 \\
Abundancia total & 102113,6 & 81512,6 & 45632,8 & 809587,5 & 1147536,3 & 146269,4 & 23669,5 \\
O. gaudichaudii & 0,0 & 0,0 & 0,52 & 0,19 & 0,20 & 0,10 & 0,23 \\
P. maculata & 38,2 & 1604,3 & 0,0 & 1082,3 & 763,9 & 916,7 & 541,1 \\
E. braziliensis & 76,4 & 3208,6 & 687,5 & 21072,1 & 4615,5 & 687,5 & 1591,5 \\
E. analoga & 101362,4 & 74917,2 & 44334,1 & 783486,1 & 1134676,6 & 140387,1 & 3558,7 \\
L. chilensis & 0,0 & 0,0 & 0,0 & 318,3 & 3819,7 & 891,3 & 10695,2 \\
B. picta & 0,0 & 0,0 & 0,0 & 509,3 & 0,0 & 0,0 & 0,0 \\
Glyceridae & 318,3 & 1782,5 & 101,9 & 2928,4 & 1114,1 & 2049,9 & 1935,3 \\
Spionidae & 318,3 & 0,0 & 0,0 & 191,0 & 636,6 & 0,0 & 668,4 \\
Nephtyidae & 0,0 & 0,0 & 509,3 & 0,0 & 1273,2 & 445,6 & 4010,7 \\
Orbiniidae & 0,0 & 0,0 & 0,0 & 0,0 & 636,6 & 891,3 & 668,4 \\
\hline
\end{tabular}



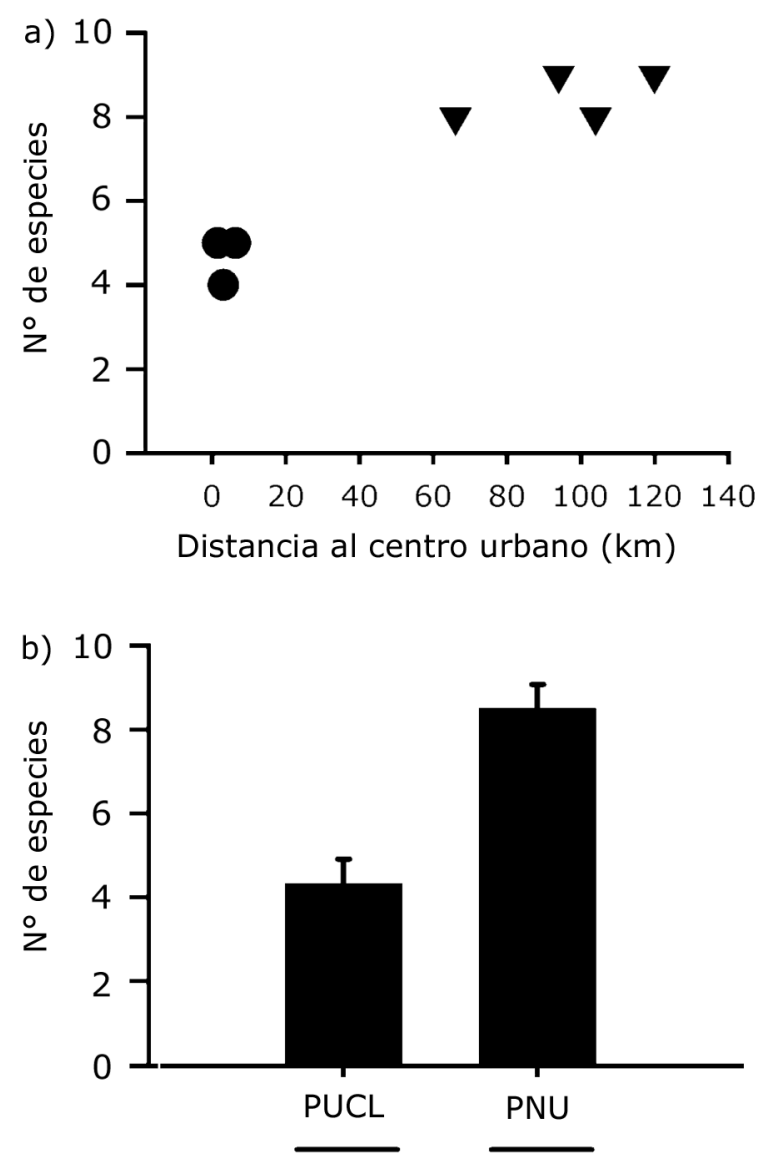

Figura 5. a) Riqueza especifica de la macroinfauna de las playas estudiadas en relación a distancias al centro urbano de Iquique. Los círculos negros representan a playas urbanas donde se realiza limpieza artificial de las mismas (Cavancha, Playa Brava y Huayquique), a la vez que los triángulos negros representan a las playas ubicadas fuera del área urbana de Iquique donde no se realiza limpieza (Chauca, Chomache, Boca del Diablo y Playa Larga). b) Valores promedio (+ 1 desviación estándar) de riqueza específica en playas urbanas con limpieza artificial (PUCL) y en playas no urbanas (PNU) ubicadas fuera del radio urbano de lquique. Las líneas negras no unidas indican ausencia de diferencias significativas ( $P$ > 0,05 ) entre los grupos comparados (ver Materiales y Métodos) / a) Species richness of the macroinfauna in relation to distances of sampling sites to the urban center of Iquique. The black dots represent urban beaches with artificial grooming (Cavancha, Playa Brava and Huayquique), while the black triangles represent beaches located outside of the urban area of Iquique where no grooming is used (Chauca, Chomache, Boca del Diablo and Playa Larga). b) Mean values (+ 1 standard deviation) of species richness in urban beaches with artificial grooming (PUCL), and in non-urban beaches (PNU) located outside of the urban area of Iquique and where no grooming is used. The non-linked black lines indicate no significant differences $(P>0.05)$ among the groups compared (see Materials and Methods)
La riqueza de especies (incluyendo a $O$. gaudichaudii) presentó una correlación directa y significativa con el perfil de la playa ( $\mathrm{r}$ de Pearson $=0,93 ; P=0,003$ ), no así el tamaño promedio de la arena ( $\mathrm{r}$ de Pearson= $-0,36 ; P=$ $0,43)$; i.e., la mayor riqueza se detectó en playas con menor inclinación o más planas.

La especie más abundante en el área de estudio fue $E$. analoga (Decapoda, Anomura), con excepción de Playa Larga, donde la especie predominante fue L. chilensis (Decapoda, Anomura) (Tabla 3). La mayor abundancia de E. analoga se encontró en las playas de Chauca y Chomache; similarmente, E. braziliensis presentó sus mayores abundancias en estas playas, a la vez que la menor abundancia de este isópodo se detectó en Cavancha. Los análisis de correlación destinados a evaluar relaciones entre la abundancia total de la macroinfauna y la de las especies más representativas del área con la distancia al centro urbano de Iquique, no mostraron correlaciones significativas: $P$. maculata $(\mathrm{r}$ de Pearson= $0,17 ; P=0,72)$, E. braziliensis (rho de Spearman= 0,27; $P=$ 0,49), E. analoga (rho de Spearman $=0,0 ; P=0,97$ ) (Fig. 6), $\mathrm{y}$ abundancia total de especies (rho de Spearman=0,0; $P=0,97$ ). Los análisis efectuados (Kruskal-Wallis) para comparar la abundancia de la macroinfauna entre los 2 tipos de playas (urbanas con limpieza artificial y no urbanas sin esta perturbación), no mostraron diferencias significativas entre ambos $(P>0,05)$ (Fig. 7).

La mayor abundancia de $O$. gaudichaudii ocurrió en Huayquique, mientras que la menor se encontró en Boca del Diablo; no se encontraron madrigueras de esta especie en Cavancha y Playa Brava (Tabla 3). Los resultados de los análisis correlación llevados a cabo para relacionar las abundancias de esta especie (incluyendo las 2 playas donde esta especie estuvo ausente) con la cercanía al centro urbano de Iquique, no mostraron correlación significativa ( $\mathrm{r}$ de Pearson= 0,$048 ; P=0,92$ ) (Fig. 6). Los análisis realizados para comparar las abundancias de $O$. gaudichaudii con respecto a la zona de estudio (incluyendo las 2 playas donde esta especie estuvo ausente), no presentaron diferencias significativas entre las playas urbanas donde se realiza limpieza artificial y aquellas fuera del radio urbano (Kruskal-Wallis, $\mathrm{H}=3,52$; $P=0,063$ ) (Fig. 7).

Los diámetros promedio de las madrigueras de $O$. gaudichaudii fluctuaron entre 1,30 y 6,77 cm (Huayquique y Chauca, respectivamente) (Fig. 8a). El diámetro de las madrigueras de Huayquique fue significativamente más bajo (Kruskal-Wallis, $\mathrm{H}=132,59, P<0,0001$ ) que el estimado 


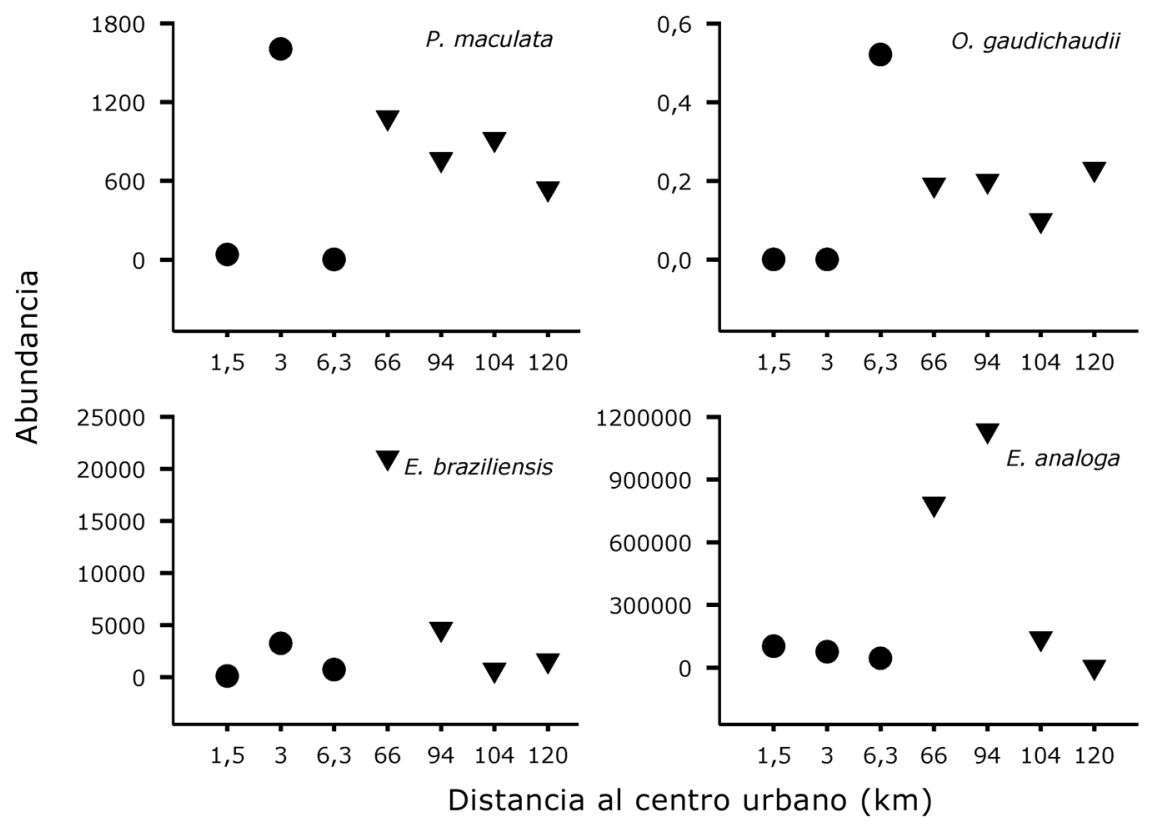

Figura 6. Abundancias promedio de Phalerisidia maculata $\left(m^{-1}\right), 0$. gaudichaudii $\left(m^{2}\right)$, Excirolana braziliensis $\left(m^{-1}\right)$ y Emerita analoga $\left(m^{-1}\right)$, en relación a distancias de los sitios de muestreo al centro urbano de lquique. Las abundancias de 0 . gaudichaudii fueron estimadas a partir de las densidades de las madrigueras de este cangrejo (ver Material y Métodos). Los círculos negros representan a playas urbanas donde se realiza limpieza artificial (Cavancha, Playa Brava y Huayquique), a la vez que los triángulos negros representan a las playas ubicadas fuera del área urbana de Iquique y donde no se realiza limpieza (Chauca, Chomache, Boca del Diablo y Playa Larga). Para ubicación latitudinal de los sitios de muestreo, ver Fig. 1 / Mean abundances of Phalerisidia maculata $\left(\mathrm{m}^{-1}\right)$, 0. gaudichaudii $\left(\mathrm{m}^{2}\right)$, Excirolana braziliensis $\left(\mathrm{m}^{-1}\right)$ and Emerita analoga $\left(\mathrm{m}^{-1}\right)$, in relation to distances of sampling sites to the urban center of Iquique. The abundances of 0 . gaudichaudii were estimated from burrow's density of this crab (see Material and Methods). The black dots represent urban beaches with artificial grooming (Cavancha, Playa Brava and Huayquique), while the black triangles represent beaches located outside of the urban area of Iquique where no grooming is used (Chauca, Chomache, Boca del Diablo and Playa Larga). For latitudinal location of sampling sites, see Fig. 1

Figura 7. Abundancias promedio (+1 desviación estándar) de Phalerisidia maculata $\left(m^{-1}\right), 0$. gaudichaudii $\left(m^{2}\right)$, Excirolana braziliensis $\left(m^{-1}\right)$ y Emerita analoga $\left(m^{-1}\right)$ en playas urbanas con limpieza artificial (PUCL), y en playas no urbanas (PNU) ubicadas fuera del radio urbano de Iquique y donde no se utiliza limpieza. Las líneas negras unidas indican ausencia de diferencias significativas $(P>0,05)$ entre los grupos comparados (ver Materiales y Métodos) / Mean abundances (+ 1 standard deviation) of Phalerisidia maculata $\left(\mathrm{m}^{-1}\right), 0$. gaudichaudii $\left(\mathrm{m}^{2}\right)$, Excirolana braziliensis $\left(\mathrm{m}^{-1}\right)$ and Emerita analoga $\left(\mathrm{m}^{-1}\right)$ in urban beaches with artificial grooming (PUCL), and in non-urban beaches (PNU) located outside of the urban area of Iquique and where no grooming is used. The linked black lines indicate no significant differences $(P>0.05)$ among the groups compared (see Materials and Methods)
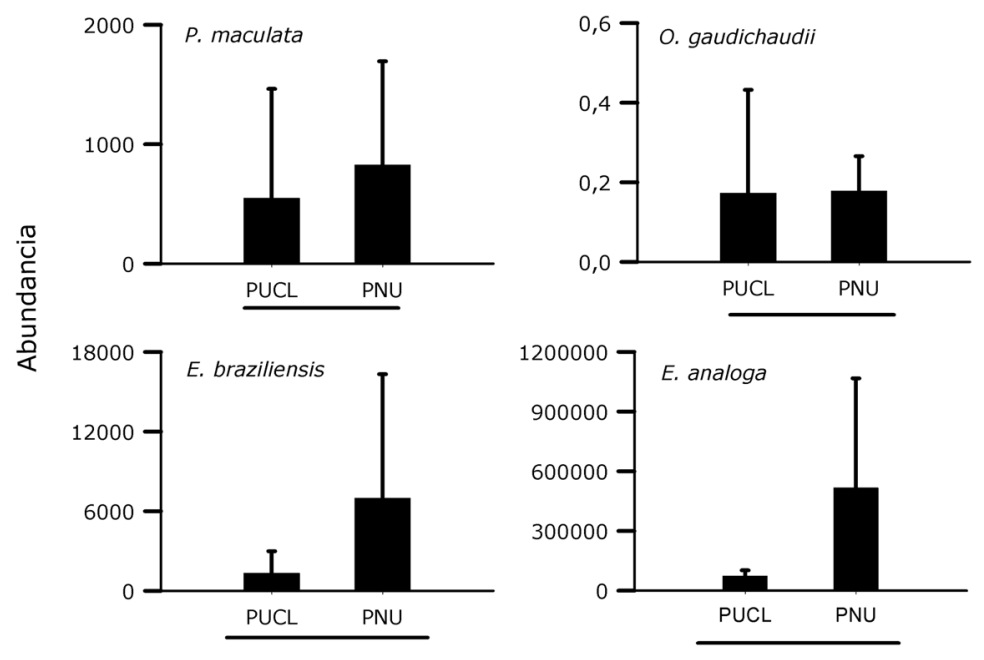
para todos los otros sitios de muestreo; en Chauca, este diámetro fue significativamente mayor $(P<0,05)$ que en Huayquique, Boca del Diablo y Playa Larga (Fig. 8a).

\section{DisTRIBUCIÓN DE OCYPODE GAUDICHAUDII}

Las madrigueras de $O$. gaudichaudii se encontraron entre el límite de marea alta más antigua y la línea de efluente, pero mayoritariamente sobre el límite de la marea alta del día. La playa en que este cangrejo presentó menor distribución espacial fue Chomache, con una distribución no mayor a $7 \mathrm{~m}$ (principalmente bajo la línea de marea alta del día) (Fig. 8b). Las playas en que O. gaudichaudii presentó una mayor distribución a lo ancho de la playa, fueron Playa Larga, con una distribución total de $20 \mathrm{~m}$ sobre la línea de marea alta del día y Chauca con $20 \mathrm{~m}$ (desde $4 \mathrm{~m}$ bajo la marea alta del día) (Fig. 8b). En Huayquique, no se registraron madrigueras de $O$. gaudichaudii sobre la línea de la marea alta del día (Fig. $8 b)$. Todas las madrigueras de este cangrejo, se ubicaron en sectores con arenas de grano medio ( $c f$. ., Folk 1980), con la excepción de los agujeros observados en Playa Larga (sustrato con arenas de tipo fino; $c f$. ., Folk 1980).

\section{RelaCiones ABUndancia de LA MACROINFAUNA Y CARACTERÍSTICAS FísiCAS DE LAS PLAYAS}

No se encontraron correlaciones significativas entre la abundancia total de la macroinfauna, la abundancia de $O$. gaudichaudii, P. maculata, E. braziliensis y E. analoga con el tamaño promedio de las partículas de arena y la pendiente de la zona que ocupan en la playa (Tabla 4).

Tabla 4. Valores de r y P, para los análisis de correlación (Pearson y Spearman) entre los parámetros biológicos y las características físicas de la playa (pendiente y tamaño del grano de arena). Los valores en negrita corresponden a aquellos resultantes de la correlación de Spearman (rho y P) / $r$ and $P$ values resulted from the correlation analyses between biological parameters and the physical characteristics of the beaches (slope and sand grain size). The values highlighted in bold are from Spearman correlation (rho and P)

\begin{tabular}{lcccccc}
\hline & \multicolumn{2}{c}{$\begin{array}{c}\text { Pendiente de } \\
\text { la playa }(1 / \mathrm{x})\end{array}$} & & \multicolumn{2}{c}{$\begin{array}{c}\text { Tamaño de la } \\
\text { partícula de } \\
\text { arena }(\mu \mathrm{m})\end{array}$} \\
\cline { 2 - 3 } & $\mathrm{r}$ & $P$ & & $\mathrm{r}$ & $P$ \\
\hline Abundancia total & & & & & \\
O. gaudichaudii & 0,47 & 0,29 & & $-0,30$ & 0,52 \\
P. maculata & $-0,53$ & 0,23 & & 0,46 & 0,30 \\
E. braziliensis & $\mathbf{0 , 2 9}$ & $\mathbf{0 , 4 9}$ & & $\mathbf{- 0 , 0 4}$ & $\mathbf{0 , 9 1}$ \\
E. analoga & $\mathbf{0 , 4 3}$ & $\mathbf{0 , 3 0}$ & & $\mathbf{0 , 2 5}$ & $\mathbf{0 , 5 5}$ \\
\hline
\end{tabular}
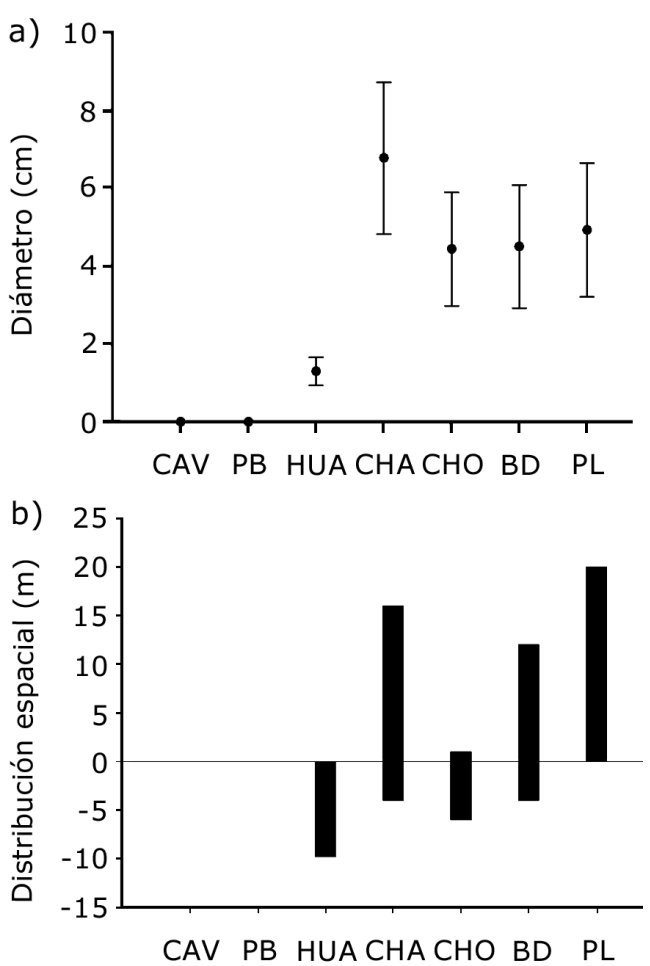

Figura 8. a) Diámetros promedio ( \pm 1 desviación estándar) de las madrigueras de Ocypode gaudichaudii. b) Distribución espacial de estas madrigueras en relación a la línea de marea alta del día de muestreo (0) en cada uno de los sitios de estudio. Las playas están ordenadas de norte a sur: $\mathrm{CAV}=$ Cavancha, $\mathrm{PB}=\mathrm{Playa}$ Brava, $\mathrm{HUA}=$ Huayquique, $\mathrm{CHA}=$ Chauca, $\mathrm{CHO}=$ Chomache, $\mathrm{BD}=\mathrm{Boca}$ del Diablo y PL= Playa Larga / a) Mean diameters ( \pm 1 standard deviation) of the burrows of Ocypode gaudichaudii. b) Spatial distribution of these burrows in relation to the high tide line of the sampling day (0) at each study sites. The beaches are ordered southward: CAV= Cavancha, $\mathrm{PB}=$ Playa Brava, $\mathrm{HUA}=$ Huayquique, $\mathrm{CHA}=$ Chauca, $\mathrm{CHO}=$ Chomache, $\mathrm{BD}=$ Boca del Diablo and $\mathrm{PL}=$ Playa Larga

\section{CONTENIdo DE Basura}

La variabilidad espacial en los contenidos de basura en cada sitio, muestra que las playas con menor cantidad de basura, (considerando los 3 niveles estudiados), fueron Cavancha, Playa Brava y Huayquique, mientras que las playas con mayor contenido de basura fueron Chomache, Boca del Diablo y Playa Larga (Fig. 9).

\section{Parámetros biológicos Versus contenido de basura}

Los análisis de correlación realizados para evaluar la eventual relación entre la abundancia total de la macroinfauna y de las especies más representativas del área con el contenido de basura no mostraron correlaciones significativas: abundancia total de la 


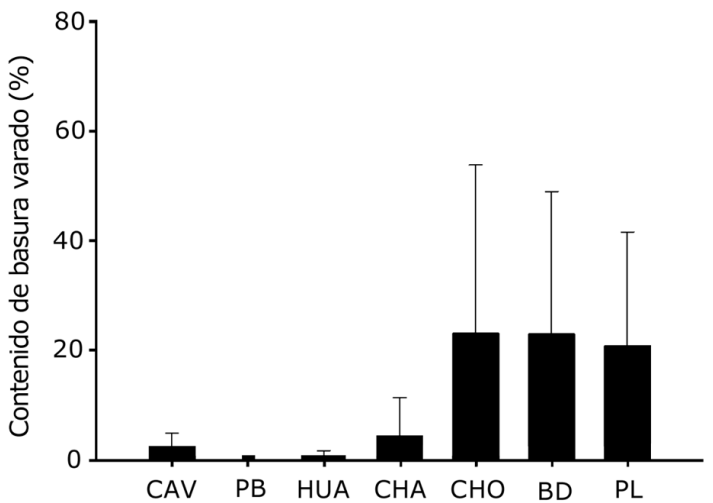

Figura 9. Porcentaje promedio (+ 1 desviación estándar) del contenido de basura varado a lo ancho de cada playa; se consideran en las estimaciones los niveles de la marea alta más antigua, marea alta del día y línea de efluente (ver Materiales y Métodos). Las playas están ordenadas de norte a sur: $\mathrm{CAV}=$ Cavancha, $\mathrm{PB}=\mathrm{Playa}$ Brava, $\mathrm{HUA}=$ Huayquique, $\mathrm{CHA}=$ Chauca, $\mathrm{CHO}=$ Chomache, $\mathrm{BD}=\mathrm{Boca}$ del Diablo y PL= Playa Larga / Mean percentage (+ 1 standard deviation) of debris stranded across shore of each beach. Percentages based in estimations at the oldest high tide line, high tide line of the sampling day and effluent line (see Materials and Methods). The beaches are ordered southward: CAV= Cavancha, $\mathrm{PB}=\mathrm{Playa}$ Brava, $\mathrm{HUA}=$ Huayquique, $\mathrm{CHA}=$ Chauca, $\mathrm{CHO}=$ Chomache, $\mathrm{BD}=$ Boca del Diablo and $\mathrm{PL}=$ Playa Larga

macroinfauna (rho de Spearman $=0,54 ; P=0,18$ ), . maculata (rho de Spearman=0,0; $P=0,97$ ), $O$. gaudichaudii (rho de Spearman $=0,25 ; \quad P=0,55), E$. braziliensis (rho de Spearman $=0,22 ; P=0,6)$ y $E$. analoga (rho de Spearman= $0,54 ; P=0,18)$. Por el contrario, la riqueza de especies estuvo significativamente correlacionada con el contenido de basura en cada sitio estudiado (rho de Spearman=0,9; $P=2 \times 10^{-7}$ ) i.e., mayor riqueza en lugares con mayor contenido de basura.

\section{Discusión}

Los resultados de este estudio muestran que la riqueza de especies de la macroinfauna intermareal de las playas arenosas de la costa de Iquique, fue significativamente más baja en sitios ubicados dentro del centro urbano de la ciudad ( $c a ., 20^{\circ} \mathrm{S}$ ), ahí donde se realiza extracción de la basura depositada en los niveles superiores de los mismos. Más aún, en esos sitios no se detectó la presencia del decápodo braquiuro $O$. gaudichaudii, una de las especies más conspicuas en las playas arenosas de la costa del norte de Chile. Por el contrario, no se encontraron diferencias al comparar las abundancias poblacionales de las otras 2 especies más comunes en el área de estudio: el isópodo cirolánido E. braziliensis y el decápodo anomuro E. analoga. La dominancia numérica de esas especies en las playas arenosas de la costa de Iquique (independiente del tipo de playa), fue similar a la encontrada por Jaramillo (1987), aproximadamente 37 años atrás.

La relación riqueza especifica de la macroinfauna versus cercanía al centro urbano de Iquique, también se observó al comparar este parámetro comunitario con el contenido de basura depositada en las playas de la costa de Iquique; i.e., la menor riqueza especifica ocurrió en playas con un menor contenido de basura (Cavancha, Playa Brava y Huayquique), situación probablemente asociada a que esas playas son limpiadas periódicamente de modo artificial, lo que resulta en perturbación del ordenamiento natural del sedimento y pérdida de microhabitat para la macroinfauna intermareal. Esto sugiere que la perturbación por efecto de la limpieza periódica de la basura depositada en las playas arenosas, es un factor relevante al evaluar el efecto de tal actividad sobre la macroinfauna intermareal de playas arenosas. No obstante los resultados de este estudio, hay que destacar que aun cuando los resultados del mismo permiten concluir que la riqueza especifica de la macroinfauna intermareal de las playas arenosas de la costa de Iquique es afectada significativamente por la perturbación por limpieza artificial, no se ha medido otro tipo de perturbación que pudiera también afectar a la macroinfauna. Es el caso del eventual efecto de la perturbación mecánica producida por la gente sobre las capas superficiales de las playas urbanas; tal efecto puede sumarse al de la limpieza artificial de basura, ya que es justamente en estas playas donde el número de personas que acude a este tipo de ambiente litoral es mayor. Es interesante señalar aquí los resultados de Moffett et al. (1998), quienes demostraron en playas de Sudáfrica (costa del Cabo), que la perturbación mecánica producida por el deambular de la gente tuvo un efecto negativo sobre los bivalvos Donax serra (Röding, 1798) y D. sordidus (Hanley, 1845) y el isópodo Eurydice longicornis (Studer, 1883).

Resultados similares a los encontrados en este estudio, han sido mencionados para diversos litorales. Colombini et al. (2003) concluyeron que la perturbación por limpieza artificial en playas arenosas de diferentes costas, causa impactos significativos en la macroinfauna, disminuyendo la riqueza y abundancia de especies. Similarmente, Dugan et al. (2003) encontraron una relación directa entre esos parámetros comunitarios y la biomasa de macroalgas varadas en playas de la costa de California. Vieira et al. (2012) compararon sectores urbanos y no urbanos en 2 
playas arenosas del sur de Brasil (Paraná y Santa Catarina), encontrando un número significativamente más bajo de especies en el sector urbano de la playa con el mayor número de visitantes, situación atribuida por estos autores a la perturbación mecánica del sedimento en ese sitio, producto de la presión antrópica a la que se ve sometido. Gilburn (2012), estudió el efecto de la limpieza de playas en aproximadamente 60 sitios en la costa de Escocia (UK), encontrando una baja riqueza y abundancia de organismos en las playas en que se realiza este tipo de actividad.

En este estudio, también se hipotetizó que la abundancia total de la macroinfauna y la abundancia de las especies características de las playas arenosas de la costa de Iquique, sería menor en playas urbanas que en aquellas alejadas del radio urbano. Los presentes resultados no muestran diferencias significativas en las abundancias de P. maculata, E. braziliensis y E. analoga al comparar playas urbanas y no urbanas, lo que difiere de los resultados de Vieira et al. (2012) quienes encontraron en playas de Brasil, relaciones negativas entre la abundancia de las especies y la cantidad de personas que accede a esas playas. Veloso et al. (2011) al estudiar las presiones antrópicas sobre el isópodo $E$. braziliensis en playas de Brasil, encontraron un efecto negativo sobre estas en las sitios donde el hombre ejerce una mayor perturbación. Por otra parte, Schierding et al. (2011) encontraron que playas arenosas del norte de Alemania (costa del Mar Báltico) ocupadas por muchos visitantes, presentan menor riqueza de especies que sitios con menor afluencia.

Diversos estudios han encontrado negativos efectos sobre poblaciones de cangrejos del genero Ocypode como consecuencia de perturbaciones causadas por turistas. Barros (2001) y Schlacher et al. (2007) en playas de Australia con $O$. cordimanus y $O$. ceratophthalma; Blankesteyn (2006) y Neves \& Bemvenuti (2006) y Magalhães et al. (2009), en playas de Brasil con $O$. quadrata (Fabricius, 1787). En este estudio, no se detectó a $O$. gaudichaudii en dos playas urbanas donde se realiza limpieza artificial (Cavancha y Playa Brava); sin embargo, hay que tener presente que las estimaciones de abundancia realizadas para este cangrejo fueron indirectas, ya que estuvieron basadas en el número de madrigueras y no en estimaciones reales. Aun así, consideramos que tal como ha sido demostrado en estudios anteriores (Turra et al. 2005, Tureli et al. 2009), la abundancia de madrigueras es un indicador apropiado de la abundancia de este cangrejo. También es importante mencionar que las 2 playas urbanas en donde no se encontraron madrigueras de $O$. gaudichaudii (Cavancha y Playa Brava), son las más cercanas a los centros urbanos de Iquique, a diferencia de la playa de Huayquique, donde se observaron madrigueras de este cangrejo e igualmente se realiza limpieza artificial de la basura depositada en la playa.

El tamaño de las madrigueras de $O$. gaudichaudii en esta última playa - el único sitio urbano en que se encontró esta especie - fue significativamente menor al de las madrigueras que ocurren en las playas ubicadas fuera del radio urbano, lo que podría ser producto de la perturbación mecánica producida por los visitantes y que resultaría en la remoción de la matriz sedimentaria. Esta conclusión es similar a la Aheto et al. (2011) para Ocypode spp. en playas de Ghana; i.e., el tamaño de las madrigueras de estos cangrejos es un buen indicador del impacto humano, posiblemente mejor que la estimación de su abundancia. Las madrigueras de $O$. gaudichaudii estudiadas en la costa de Iquique, se ubicaron preferentemente alrededor del nivel de la marea alta del día. Esto fue similar a lo observado aproximadamente 17 años atrás por Quijón et al. (2001) en playas de la misma zona y a lo estudiado para $O$. quadrata en la costa de Brasil (Turra et al. 2005) y para O. cursor (Linnaeus, 1758) en la costa de Turquía (Tureli et al. 2009). Turra et al. (2005) y Tureli et al. (2009), también encontraron una correlación entre el tamaño del cangrejo con su cercanía al mar (i.e., las madrigueras más pequeñas se ubican más cerca del nivel de marea baja). En el presente estudio no se midió la distancia de cada agujero a tal límite mareal; sin embargo, la observación de los tamaños y la distribución promedio de las madrigueras de este cangrejo, permite corroborar que la tendencia se mantiene. Por ejemplo, en Huayquique y Chomache - playas donde se observaron las madrigueras con los diámetros más pequeños - los agujeros de enterramiento de $O$. gaudichaudii ocurrieron mayoritariamente bajo el nivel de la marea alta del día. Por el contrario, en Chauca y Playa Larga - las playas con las madrigueras de mayores diámetros - la ubicación de los agujeros de enterramiento de $O$. gaudichaudii se encontró preferentemente sobre el límite de la marea alta del día, lo que confirma la relación entre el tamaño de $O$. gaudichaudii y la ubicación de esta especie a lo ancho de la playa.

Turra et al. (2005) plantearon evaluar la relación entre la distribución de Ocypode quadrata con parámetros físicos como el tamaño de la partícula de arena y la pendiente de la playa en las costa de Brasil. En este 
estudio, al igual que en el efectuado por Tureli et al. (2009), se encontró que $O$. gaudichaudii ocurre principalmente en arenas de tamaño medio ( $c f$., Folk 1980). Aun cuando no se encontraron relaciones entre la abundancia de las madrigueras con la pendiente y ancho del intermareal, en la playa con un intermareal más ancho (Playa Larga), $O$. gaudichaudii se encontró solo sobre el límite de la marea alta del día y en aquella con el menor ancho (Huayquique), solo se la encontró bajo este límite. Lo anterior permite inferir que existe una relación entre la distribución de esta especie a lo ancho del intermareal con el ancho del sitio donde se encuentra.

En conclusión, en este estudio se encontró un efecto negativo de la perturbación del sedimento (producto de la limpieza artificial de las playas) sobre la riqueza de especies de la macroinfauna que habita las playas arenosas de la costa de Iquique, siendo las especies afectadas aquellas que ocupan primariamente los niveles superiores (O. gaudichaudii) y medios (poliquetos) de la zona intermareal. En este sentido, la especie más afectada es el cangrejo Ocypode gaudichaudii, la cual no fue observada en 2 playas donde ocurre limpieza artificial de las mismas (Cavancha y Playa Brava). Es interesante señalar que cuando era ausente o escaza ese tipo de perturbación, esta especie ocurría en playas donde hoy no ocurre o es escasa ( $c f$., Jaramillo 1987). Esto muestra que el hábitat natural de esta especie -los niveles superiores de las playas de arena- están constantemente amenazado por el desarrollo costero, cosa no trivial cuando la distribución de esta especie en Chile está restringida a la costa comprendida aproximadamente entre Arica y la Península de Mejillones (obs. pers.) y considerando que la función y rol de los cangrejos de este género en el ecosistema de playas arenosas es relevante dada la capacidad de transferir energía, ya sea depredando y consumiendo carroña (Wolcot 1978) o extrayendo microalgas de las arenas de las playas (Robertson \& Pfeiffer 1981). Más aún, diversos autores han mostrado que las especies de Ocypode son indicadores apropiados de las perturbaciones humanas producidas en las playas arenosas: e.g., Blanckensteyn (2006), Neves \& Bemvenuti (2006), Araujo et al. (2008), Moss \& McPhee (2006), Schlacher et al. (2007), Barros (2001) y Peterson et al. (2000). De ahí la necesidad de realizar estudios - entre otros aspectos, con mayor frecuencia de muestreo a la utilizada en este trabajo - y complementar más información sobre el estado de conservación de esta importante especie de las playas arenosas de la costa del norte de Chile.
Los antecedentes anteriores permiten considerar a los cangrejos del genero Ocypode como organismos indicadores e icónicos de perturbaciones ambientales en playas arenosas de diferentes litorales (Harris et al. 2014). Consecuentemente, es necesario realizar investigaciones que permitan por ejemplo, conocer las respuestas conductuales de Ocypode a diferentes magnitudes de cambios ambientales, tal como ha sido sugerido por Scapini et al. (2014), para otras especies características de playas arenosas.

Anteriormente se mencionó que podría haber otros factores afectando la estructura comunitaria de las playas arenosas estudiadas. Entre tales factores, podría estar por ejemplo el efecto de la depredación por aves playeras - específicamente aquellas que consumen E. analoga efecto que podría ser evaluado mediante la realización de experimentos naturales; e.g., evaluación de abundancias de este crustáceo en playas con diferentes abundancias de aves, pero similares en cuanto a actividades antrópicas (e.g., sin extracción de basura). Otro experimento natural sería por ejemplo, comparar el número de madrigueras de $O$. gaudichaudii (especie icónica del área; $c f$., Harris et al. 2014) en playas con similares cantidades de basura, pero con diferentes facilidades de acceso a vehículos que perturban los niveles superiores de las mismas, justamente aquellos donde habita este crustáceo. Estudios de este tipo, permitirían avanzar en el diseño y puesta en acción de estrategias de conservación de un hábitat litoral de amplia representación en todas las costas del mundo (Brown 2001).

\section{Agradecimientos}

Este estudio fue financiado por el Proyecto FONDECYT 1121043 dirigido por Eduardo Jaramillo. Los autores agradecen la ayuda en terreno de los biólogos marinos Roland Sánchez y Carlos Velásquez.

\section{LITERATURA CITADA}

Aheto D, C Asare, E Mensah \& J Aggrey-Fynn. 2011. Rapid assessment of anthropogenic impacts of exposed sandy beaches in Ghana using ghost crabs (Ocypode spp.) as ecological indicators. Momona Ethiopian Journal of Science 3: 93-103.

Araujo CCV, DM Rosal \& JM Fernandes. 2008. Densidade e distribuição espacial do caranguejo Ocypode quadrata (Fabricius, 1787) (Crustacea, Ocypodidae) em três praias arenosas do Espírito Santo, Brasil. Revista Biotemas 21: 73-80. 
Barros F. 2001. Ghost crabs as a tool for rapid assessment of human impacts on exposed sandy beaches. Biological Conservation 97: 399-404.

Blankensteyn A. 2006. O uso do caranguejo maria-farinha Ocypode quadrata (Fabricius) (Crustacea, Ocypodidae) como indicador de impactos antropogênicos em praias arenosas da Ilha de Santa Catarina, Santa Catarina, Brasil. Revista Brasileira de Zoologia 23: 870-876.

Brown AC. 2001. Biology of sandy beaches. In: Steele JH, SA Thorpe \& KK Turekian (eds). Encyclopedia of Ocean Sciences 5: 2496-2504. Academic Press, London.

Colombini I, L Chelazzi, R Gibson \& R Atkinson. 2003. Influence of marine allochthonous input on sandy beach communities. Oceanography and Marine Biology: An Annual Review 41: 115-159.

Defeo O, A McLachlan, D Schoeman, T Schlacher, J Dugan, A Jones, M Lastra \& F Scapini. 2009. Threats to sandy beach ecosystems: a review. Estuarine, Coastal and Shelf Science 81: 1-12.

Dugan J, D Hubbard, M McCrary \& M Pierson. 2003. The response of macrofauna communities and shorebirds to macrophyte wrack subsidies on exposed sandy beaches of southern California. Estuarine, Coastal and Shelf Science 58: $25-40$

Emery KO. 1938. Rapid method of mechanical analysis of sands. Journal of Sedimentary Petrology 8: 105-111.

Emery KO. 1961. A simple method of measuring beach profiles. Limnology and Oceanography 6: 90-93.

Folk R. 1980. Petrology of sedimentary rocks, 182 pp. Hemphill Publishing, Texas.

Gilburn A. 2012. Mechanical grooming and beach award status are associated with low strandline biodiversity in Scotland. Estuarine, Coastal and Shelf Science 107: 81-88.

Harris L, N Ronel, S Holness, K Sink \& D Schoeman. 2014. Setting conservation targets for sandy beach ecosystems. Estuarine, Coastal and Shelf Science 150: 4557.

Jaramillo E. 1987. Sandy beach macroinfauna from the Chilean coast: Zonation patterns and zoogeography. Vie et Milieu 37: $165-174$.

Jaramillo E, C Duarte \& H Contreras. 2000. Sandy beach macroinfauna from the coast of Ancud, Isla de Chiloe, Southern Chile. Revista Chilena de Historia Natural 73: 771-786.

Kohler K \& S Gill. 2006. Coral Point Count with Excel extensions (CPCe): A Visual Basic program for the determination of coral and substrate coverage using random point count methodology. Computers \& Geosciences 32: 1259-1269.

Kruskal WH \& WA Wallis. 1952. Use of ranks in one-criterion variance analysis. Journal of the American Statistical Association 47: 583-621.
Magalhães W, J Lima, F Barros \& J Dominguez. 2009. Is Ocypode quadrata (Fabricius, 1787) a useful tool for exposed sandy beaches management in Bahia State (Northeast Brazil)? Brazilian Journal of Oceanography 57: 153-155.

Moffett M, A McLachlan, P Winter \& A De Ruyck. 1998. Impact of trampling on sandy beach macrofauna. Journal of Coastal Conservation 4: 87-90.

Moss D \& D McPhee. 2006. The impacts of recreational fourwheel driving on the abundance of the ghost crab (Ocypode cordimanus) on a subtropical sandy beach in SE Queensland. Coastal Management 34: 133-140.

Nel R, EE Campbell, L Harris, L Hauser, DS Schoeman, A McLachlan, DR du Preez, K Bezuidenhout, TA Schacher. 2014. The status of sandy beach science: Past trends, progress, and possible futures. Estuarine, Coastal and Shelf Science 150: 1-10.

Neves F \& C Bemvenuti. 2006. The ghost crab Ocypode quadrata (Fabricius, 1787) as a potential indicator of anthropic impact along the Rio Grande do Sul coast, Brazil. Biological Conservation 133: 431-435.

Peterson CH, DH Hickerson \& GG Johnson. 2000. Shortterm consequences of nourishment and bulldozing on the dominant large invertebrates of a sandy beach. Journal of Coastal Research 16(2): 368-378.

Quijón P, E Jaramillo \& H Contreras. 2001. Distribution and habitat structure of Ocypode gaudichaudii $\mathrm{H}$. Milne Edwards \& Lucas, 1843, in sandy beaches of northern Chile. Crustaceana 74: 91-103.

Robertson JR \& PJ William. 1981. Deposit-feeding by the ghost crab Ocypode quadrata (Fabricius). Journal of Experimental Marine Biology and Ecology 56: 165-177.

Salvat B. 1964. Les conditions hydrodynamiques interstitielle des sediment meubles intertidaux et la repartition verticale de la jenne endogee. Comptes Rendus de l'Academie des Sciences, Paris 259: 1567-1579.

Scapini F. 2014. Behaviour of mobile macrofauna is a key factor in beach ecology as response to rapid environmental changes. Estuarine, Coastal and Shelf Science 150: 36-44.

Schierding M, S Vahder, L Dau \& U Irmler. 2011. Impacts on biodiversity at Baltic Sea beaches. Biodiversity and Conservation 20: 1973-1985.

Schlacher T, L Thompson \& S Price. 2007. Vehicles versus conservation of invertebrates on sandy beaches: mortalities inflicted by off - road vehicles on ghost crabs. Marine Ecology 28: 354-367.

Seward-Thompson B \& J Hails. 1973. An appraisal of the computation of statistical parameters in grain size analysis. Sedimentology 20: 161-169.

Tureli C, O Duysak, E Akamca \& V Kiyagi. 2009. Spatial distribution and activity pattern of the ghost crab, Ocypode cursor (L., 1758) in Yumurtalik bay, North-Eastern Mediterranean-Turkey. Journal of Animal and Veterinary Advances 8: 165-171. 
Turra A, M Gonçalves \& M Denadai. 2005. Spatial distribution of the ghost crab Ocypode quadrata in lowenergy tide-dominated sandy beaches. Journal of Natural History 39: 2163-2177.

Veloso V, G Neves \& L de Almeida-Capper. 2011. Sensitivity of a cirolanid isopod to human pressure. Ecological Indicators 11: 782-788.

Veloso V, E Silva, C Caetano \& R Cardoso. 2006. Comparison between the macroinfauna of urbanized and protected beaches in Rio de Janeiro State, Brazil. Biological Conservation 127: 510-515.
Vieira J, C Borzone, L Lorenzi \& F Carvalho. 2012. Human impact on the benthic macrofauna of two beach environments with different morphodynamic characteristics in southern Brazil. Brazilian Journal of Oceanography 60: $135-148$.

Wolcott TG. 1978. Ecological role of ghost crabs, Ocypode quadrata (Fabricius) on an ocean beach: Scavengers or predators? Journal of Experimental Marine Biology and Ecology 31: 67-82.

Recibido el 8 de octubre de 2014 y aceptado el 23 de marzo de 2015

Editor Asociado: Mauricio Landaeta D. 